\title{
Correlation between leukocytospermia and oxidative stress in male partners of infertile couples with leukocytospermia
}

\author{
Richa Aggarwal ${ }^{1 *}$, Manju Puri', Rima Dada ${ }^{2}$, Gyan Saurabh $^{3}$
}

\author{
${ }^{1}$ Department of Obstetrics \& Gynaecology, Lady Hardinge Medical College, New Delhi, Delhi, India \\ ${ }^{2}$ Department of Anatomy, All India Institute of Medical Sciences, New Delhi, Delhi, India \\ ${ }^{3}$ Department of Surgery, Lady Hardinge Medical College, New Delhi, Delhi, India
}

Received: 15 December 2014

Accepted: 11 January 2015

\author{
*Correspondence: \\ Dr. Richa Aggarwal, \\ E-mail: drrichaaggarwal@yahoo.co.in
}

Copyright: (C) the author(s), publisher and licensee Medip Academy. This is an open-access article distributed under the terms of the Creative Commons Attribution Non-Commercial License, which permits unrestricted non-commercial use, distribution, and reproduction in any medium, provided the original work is properly cited.

\begin{abstract}
Background: The last century has seen rapid advances in the diagnosis and management of male infertility but still the cause eludes us in a majority of cases. The World Health Organization (WHO) has defined leukocytospermia as $>1$ million $\mathrm{WBC} / \mathrm{mL}$ of semen. However, the clinical significance of increased leukocyte infiltration in semen, that is, leukocytospermia, is currently a subject of controversy. Evidence from several recent studies indicates that leukocytospermia could significantly contribute to male infertility. Of the many causes of male infertility, oxidative stress has been identified as one factor that affects fertility status and thus, has been extensively studied in recent years. A study was conducted to evaluate the relationship between leukocytospermia and oxidative stress.

Methods: 88 men with leukocytospermia were included in the study. Semen parameters, no. of pus cells present and oxidative stress were noted. The correlation between leukocytospermia and ROS level was found using Pearson correlation coefficient.

Results: Significant positive correlation $(\mathrm{r}=0.882, \mathrm{P}<0.001)$ was observed between leukocytospermia and oxidative stress suggesting leukocytes are the main source of Reactive Oxygen Species (ROS) in semen.

Conclusions: Significant positive correlation between oxidative stress and leukocytospermia was found in this study suggesting the need of further robust clinical trials to evaluate the role of antioxidants in improving the fertility outcome in infertile men with pyospermia.
\end{abstract}

Keywords: Leukocytospermia, Oxidative stress, Male infertility, Reactive oxygen species

\section{INTRODUCTION}

Infertility is defined as the failure of conception after at least 12 months of unprotected intercourse. ${ }^{1}$ Infertility is a worldwide problem and approximately $8-10 \%$ of couples within reproductive age group are infertile. ${ }^{2}$ It is estimated that globally 60-80 million couples suffer from infertility every year, of which probably $15-20$ million are in India alone. ${ }^{3}$ Male factor is the sole cause of infertility in approximately $20 \%$ of infertile couples and contributory in $30-40 \%$ of couples, accounting for infertility in overall $50 \%$ of couples. ${ }^{4}$ Male infertility management has grown at a slower pace as compared to that of female infertility. The last century has seen rapid advances in the diagnosis and management of male infertility but still the cause eludes us in a majority of cases. Though there has been a lot of research in this area there is a continued need for more such endeavors to unravel the mystery of male infertility.

While evaluating an infertile couple, the World Health Organization (WHO) recommends performing a semen analysis on the male partner. The White Blood Cell (WBC) count in semen is one of the classical measures of semen quality. The etiology of leukocytospermia in 
healthy asymptomatic men is not fully understood. Pyospermia has multifactorial causes, such as infection, inflammation and autoimmunity. The etiology can be classified into several categories: presence of defective sperms, varicocele, chronic prostatitis, smoking, drug abuse like marijuana (social causes), alcohol, exposure to irritants and toxins, use of vaginal products by partner during sexual activity, prolonged abstinence, vasovasotomy, clomiphene citrate therapy, urethroplasty, genital infections like Chlamydia trachomatis, Gardenella vaginalis and Ureaplasma urealyticum, genital infections in patients' sexual partners. ${ }^{5}$ The World Health Organization (WHO) internationally standardizes and defines pyospermia as $1 \times 10^{6} \mathrm{WBCs} / \mathrm{mL}$ semen as diagnosed by measuring either peroxidase or by immunohistological detection methods. ${ }^{6}$ However, the clinical significance of increased leukocyte infiltration in semen, that is, leukocytospermia, is currently a subject of controversy. $^{7}$ Evidence from several recent studies indicates that leukocytospermia could significantly contribute to male infertility by decreasing sperm motility and fertilizing ability. ${ }^{8}$

Of the many causes of male infertility, oxidative stress has been identified as one factor that affects fertility status and thus, has been extensively studied in recent years. Of all the different types of cells present in semen, leukocytes and spermatozoa have been shown to be the two main sources of ROS. ${ }^{9}$ Spermatozoa, like any other aerobic cell, are constantly facing the "oxygenparadox". ${ }^{10}$ Low levels of ROS have been shown to be essential for fertilization, acrosome reaction, hyperactivation, motility, and capacitation. ${ }^{11,12}$ Conversely, high levels of breakdown products of oxygen such as ROS can be detrimental to cell function and survival. ${ }^{10}$ All cellular components, including lipids, proteins, nucleic acids, and sugars are potential targets of oxidative stress. Spermatozoa are particularly susceptible to damage induced by ROS because their plasma membranes contain large quantities of polyunsaturated fatty acids $^{13}$ and their cytoplasm contains low concentrations of scavenging enzymes. ${ }^{14}$ In addition, the intracellular antioxidant enzymes cannot protect the plasma membrane that surrounds the acrosome and the tail, forcing spermatozoa to supplement their limited intrinsic antioxidant defenses by dependence on the protection afforded by the seminal plasma that bathes these cells. ${ }^{15}$ Oxidative stress develops when levels of ROS production by leukocytes, spermatozoa, or both, become high enough to overwhelm all antioxidant strategies, resulting in lipid peroxidation which leads to impairment of sperm motility and loss of fertilizing potential. ${ }^{16}$ Oxidative stress is also associated with high frequencies of single and double strand DNA breaks resulting in impaired semen quality. ${ }^{17}$

The purpose of this study is to evaluate the relationship between oxidative stress and leukocytospermia in male partners of infertile couples with pyospermia.

\section{METHODS}

This study was conducted in the department of obstetrics and gynaecology, Lady Hardinge medical college and Smt. Sucheta Kriplani hospital, New Delhi for a period of one year.

\section{Study design}

Observational study.

\section{Study population}

All the male partners of the infertile couples attending the infertility clinic of our hospital were subjected to a detailed history, examination and investigations. Detailed history regarding duration of infertility, coital frequency, any sexual dysfunction, previous medical or surgical illness, occupational history, life style factors was taken. Physical examination was performed by a urologist to exclude cases with known factors such as varicocele, cryptorchidism and endocrine disorders. Men with history of tobacco chewing/smoking, alcohol consumption, prolonged thermal/radiation exposure, prolonged medical illness were excluded from the study.

\section{Sample size}

88 male partners with semen analysis showing the presence of significant leukocytospermia (WBC $>1 \times 10^{6} / \mathrm{mL}$ ) were included in the study.

Semen analysis and oxidative stress (ROS) measurement was done in all the samples.

\section{Semen collection and semen analysis}

Semen samples were collected in a sterile plastic container after sexual abstinence of 3-5 days. All the samples were examined within one hour of collection. Each sample was incubated at room temperature to allow liquefaction. Semen analysis was performed according to the guidelines of the WHO. The gross appearance, volume, viscosity and $\mathrm{pH}$ semen were noted. A wet mount of semen sample was prepared and microscopic examination was done to measure sperm concentration, sperm motility and sperm vitality. Sperm concentration was determined with an improved Neubauer counting chamber after appropriate dilution. Sperm morphology, specific sperm defects, leukocyte count were determined after staining the smear with Papincolaou stain.

\section{Estimation of ROS by chemiluminescence assay}

Levels of ROS were measured in a fresh semen sample using a chemiluminescence assay. Four hundred microlitre aliquots of the sample were allowed to run in the luminometer to assess basal ROS levels. Ten microlitre of luminol (5-amino-2,3,-dihydro-1,4phthalazinedione) prepared as $5 \mathrm{mM}$ stock in dimethyl 
sulphoxide, was added to the mixture and served as a probe. A negative control was prepared by adding $10 \mu \mathrm{l}$ of $5 \mathrm{mM}$ luminol to $400 \mu \mathrm{l}$ of tap water. The reaction of luminol with ROS results in production of a light signal that is converted to an electrical signal (photon) by a luminometer. Levels of ROS were assessed by measuring the luminol-dependent chemiluminescence with the luminometer for 10 minutes. The results were expressed as RLU/million sperms/second.

\section{Statistical analysis}

All the semen parameters, age, duration of infertility, no. of pus cells and ROS values were expressed as mean (minimum range, maximum range). The correlation between leukocytospermia and ROS level was found using Pearson correlation coefficient.

\section{RESULTS}

Semen samples of the male partners of infertile couples attending our O.P.D. were examined and samples showing significant leukocytospermia were subjected to ROS assessment. 88 semen samples were included in this study. Semen profile of the study sample is shown in Table 1.

Significant correlation was observed between leukocytospermia and oxidative stress $(\mathrm{r}=0.882, \mathrm{P}$ $<0.001)$ as can be seen from the Figure 1 .

Table 1: Semen profile of the study group.

\begin{tabular}{|c|c|c|c|c|c|c|c|}
\hline & Age (years) & $\begin{array}{l}\text { Duration } \\
\text { of infertility } \\
\text { (years) }\end{array}$ & $\begin{array}{l}\text { Total sperm } \\
\text { concentration } \\
\text { (Million/mL) }\end{array}$ & $\%$ Motility & \%Morphology & $\begin{array}{l}\text { No. of pus } \\
\text { cells } \\
\left(\times 10^{6} / \mathrm{mL}\right)\end{array}$ & $\begin{array}{l}\text { Oxidative stress } \\
\text { (RLU/Million } \\
\text { sperms/second) }\end{array}$ \\
\hline Mean \pm SD & $29.23 \pm 4.65$ & $5.05 \pm 2.79$ & $60.40 \pm 33.58$ & $46.19 \pm 17.46$ & $43.87 \pm 17.89$ & $2.28 \pm 1.26$ & $79.72 \pm 133.96$ \\
\hline
\end{tabular}

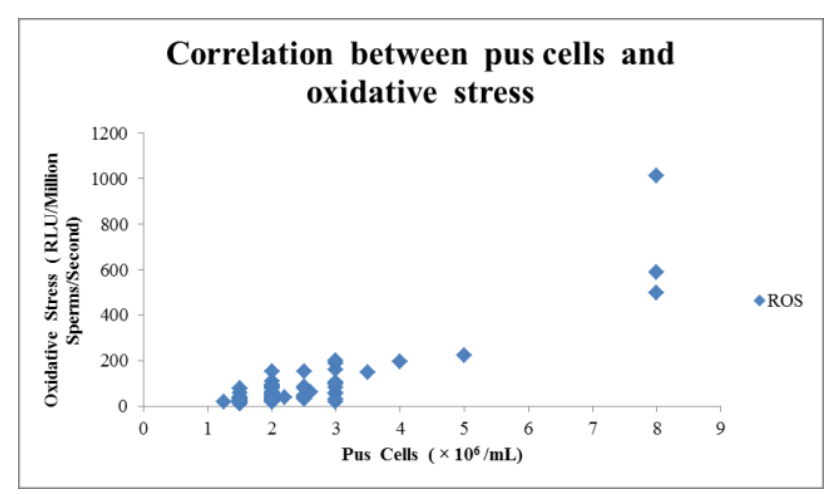

Figure 1: Correlation between pus cells and oxidative stress.

Table 2: Correlation coefficient between sperm parameters and ROS.

\begin{tabular}{|llll|}
$\begin{array}{l}\text { Total sperm } \\
\text { concentration }\end{array}$ & $\%$ Motility & $\%$ Morphology & $\begin{array}{l}\text { No. of } \\
\text { pus cells }\end{array}$ \\
\hline-0.233 & -0.303 & -0.259 & 0.882 \\
\hline
\end{tabular}

\section{DISCUSSION}

Recently, oxidative stress has become the focus of interest as a potential cause of male infertility. While both leukocytes and spermatozoa produce ROS, leukocytes have been reported to produce 1000 times more ROS as compared to spermatozoa. ${ }^{18,19}$ Besides directly producing reactive oxygen species, leukocytes may also lead to increased oxidative stress by causing adverse changes in spermatozoa. Morphologically abnormal sperms, especially those with cytoplasmic retention have been shown to generate high levels of ROS. Our study observed significant positive correlation $(\mathrm{r}=0.882, \mathrm{P}$ $<0.05$ ) between leukocytospermia and oxidative stress which is in consistence with several other studies showing that leukocytes are the major source of Reactive Oxygen Species (ROS) in semen. ${ }^{20,21}$

It is unclear from the existing literature whether the interaction between leukocytes and spermatozoa implies a direct or indirect stimulatory effect, which may enhance the capacity of spermatozoa to generate excessive ROS.

Saleh et al. found that levels of spontaneous and phenol 12-myristate13-acetate (PMA) induced ROS production by spermatozoa from infertile men with leukocytospermia was significantly higher compared with the infertile men without leukocytospermia and normal donors. A similar pattern of increased spontaneous and PMA- induced ROS production by human spermatozoa was observed when spermatozoa from nonleukocytospermic samples were incubated with peripheral blood neutrophils. They hypothesized that seminal leukocytes may play a role in stimulating excessive ROS production by spermatozoa. The mechanism of such stimulation is unclear and may be due to a direct sperm-leukocyte contact or mediated by soluble products released by the leukocytes. Leukocytospermia, in their study, was associated with a significant reduction of seminal Total Antioxidant Capacity (TAC) levels. Saleh et al. concluded in their study that spermatozoa from leukocytospermic patients 
are at a higher risk for leukocyte-mediated oxidative stress. $^{22}$

Much of the controversy centers on the best definition of pathological leukocytospermia and the correlation of WBCs with seminal oxidative stress is unclear. The WHO definition is more than $1 \times 10^{6} \mathrm{WBC} / \mathrm{mL}$ of semen. However, the minimum WBC count that impairs fertility may be lower or higher. Sharma et al. compared oxidative stress in semen samples with different leukocyte counts and determined the correlation between leukocyte counts and oxidative stress. They were unable to establish the minimum level of WBCs associated with oxidative stress because it appeared that the presence of any seminal leukocytes, no matter how few, was associated with oxidative stress. Samples with no seminal leukocytes had significantly lower ROS levels and significantly higher total antioxidant scores than samples with any seminal leukocytes, even very low levels. They concluded that oxidative stress occurs even in patients with very low seminal WBC counts (between 0 and $1 \times 10^{6} / \mathrm{mL}$ ) and rises with an increase in WBC count. ${ }^{23}$

In addition, our study observed a significant negative correlation between oxidative stress and sperm concentration, motility, and sperm morphology, reflecting a strong association of ROS with impaired semen quality. The mechanism underlying this set of findings is not clear and may indicate a causal relationship or a circumstantial reflecting a common underlying pathology. A possible explanation is that excessive amount of ROS can cause damage to the normal spermatozoa by inducing lipid peroxidation, impairing sperm motility and causing DNA damage.

However, our study has several limitations. The semen smears were not examined for spermatozoa with excessive residual cytoplasm, which may be also contributing ROS. Patients have not been followed for long enough to relate the results to the fertility outcome. Hence it is suggested that men with persistent leukocytospermia should be enrolled in further studies to define the etiology and clinical significance of leukocytospermia and develop treatment modalities to improve male infertility.

\section{CONCLUSIONS}

In the last decade, a phenomenal growth has occurred in our knowledge of male reproduction, sperm function, and development of newer diagnostic and treatment modalities for male infertility. However, despite of extended diagnostic efforts the cause of leukocytospermia cannot be established in most of the cases. The clinical significance of leukocytospermia continues to remain controversial. Recently, oxidative stress has become the focus of interest as a potential cause of male infertility and has been shown to impair sperm motility, morphology and cause DNA damage. The present study observed significant positive correlation between leukocytospermia and oxidative stress suggesting further robust clinical trials to evaluate the role of antioxidants in improving the fertility outcome infertile men with pyospermia.

\section{Funding: No funding sources}

Conflict of interest: None declared

Ethical approval: The study was approved by the institutional ethics committee

\section{REFERENCES}

1. Rowe PJ, Compaire FH, Hargereave TB. WHO manual for standard investigation and diagnosis of the infertile couple. In: Rowe PJ, Compaire FH, Hargereave TB, eds. WHO Manual. Cambridge: Cambridge University Press; 1993.

2. World Health Organization. Recent advances in medically assisted conception. WHO Tech Res Ser. 1992;2:820-2.

3. World Health Organization. The world health report. Geneva: WHO; 1996. Available at: http://www.who.int/whr/1996/en/.

4. Thonneau P, Marchand S, Tallec A, Ferial ML, Ducot B, Lansac J, et al. Incidence and main causes of infertility in a resident population (1850000) of three French regions (1988-1989). Hum Reprod. 1991;6:811-816.

5. Baratt CLR, Bolton AE, Cooke ID. Functional significance of white blood cells in the male and female reproductive tract. Hum Reprod. 1990;5:63948.

6. World Health Organization. Cervical Mucus Interaction. In: WHO, eds. WHO Laboratory Manual for the Examination of Human Sperm and Semen. 4th ed. New York, NY: Cambridge University Press; 1999: 128.

7. Thomas J, Fishel SB, Hall JA, Green S, Newton TA, Thornton SJ. Increased polymorphonuclear granulocytes in seminal plasma in relation to sperm morphology. Hum Reprod. 1997;12:2418-21.

8. Arata de Bellabarba G, Tortolero I, Villarroel V, Molina CZ, Bellabarba C, Velazquez E. Nonsperm cells in human semen and their relationship with semen parameters. Arch Androl. 2000;45:131-6.

9. Garrido N, Meseguer M, Simon C, Pellicer A, Remohi J. Pro-oxidative and anti-oxidative imbalance in human semen and its relation with male infertility. Asian J Androl. 2004;6:59-65.

10. Sies H. Strategies of antioxidant defense. Eur J Biochem. 1993;215:213-9.

11. Agarwal A, Nallella KP, Allamaneni SS, Said TM. Role of antioxidants in treatment of male infertility: an overview of the literature. Reprod Biomed Online. 2004;8:616-27.

12. Griveau JF, Le Lannou D. Reactive oxygen species and human spermatozoa: physiology and pathology. Int J Androl. 1997;20:61-9. 
13. Alvarez JG, Storey BT. Differential incorporation of fatty acids from phospholipids of human spermatozoa. Mol Reprod Dev. 1995;42:334-45.

14. Storey BT, Alvarez JG, Thompson K. Human sperm glutathione reductase activity in situ reveals limitation in the glutathione antioxidant defense system due to supply of NADPH. Mol Reprod Dev. 1998;49:400-7.

15. Iwasaki A, Gagnon C. Formation of reactive oxygen species in spermatozoa of infertile patients. Fertil Steril. 1992;57:409-16.

16. Sikka SC, Rajasekaran M, Hellstrom WJG. Role of oxidative stress and antioxidants in male infertility. $\mathrm{J}$ Androl. 1995;16:464-8.

17. Kodama H, Yamaguchi R, Fukuda J, Kasai H, Tanaka T. Increased oxidative deoxyribonucleic acid damage in the spermatozoa of infertile male patients. Fertil Steril. 1997;68:519-24.

18. Fisher H, Aitken R. Comparative analysis of the ability of precursor germ cells and epididymal spermatozoa to generate reactive oxygen metabolites. J Exp Zool. 1997;277:390-400.

19. Shekarriz M, Sharma RK, Thomas AJ Jr, Agarwal A. Positive myeloperoxidase staining (Endtz test) as an indicator of excessive reactive oxygen species formation in the semen. J Assist Reprod Genet. 1995;12:70-4.
20. Eggert-Kruse W, Bellman A, Rohr G, Tilgen W, Runnebaum B. Differentiation of round cells in semen by means of monoclonal antibodies and relationship with male infertility. Fertil Steril. 1992 Nov;58(5):1046-55.

21. Tomlinson MJ, Barrat GLR, Cooke ID. Prospective study of leukocytes and leukocyte sub-populations in semen suggests that they are not a cause of male infertility. Fertil Steril. 1993;60:1069-75.

22. Saleh RA, Agarwal A, Kandirali E, Sharma RK, Thomas AJ, Nada EA, et al. Leukocytospermia is associated with increased reactive oxygen species production by human spermatozoa. Fertil Steril. 2002;78(6):1215-24.

23. Sharma RK, Pasqualotto Fabio, Nelson DR. Relationship between seminal white blood cell counts and oxidative stress in men treated at an infertility clinic. J Androl. 2001;22:575-83.

DOI: $10.5455 / 2320-1770 . \mathrm{ijr} \operatorname{cog} 20150230$

Cite this article as: Aggarwal R, Puri M, Dada R, Saurabh G. Correlation between leukocytospermia and oxidative stress in male partners of infertile couples with leukocytospermia. Int J Reprod Contracept Obstet Gynecol 2015;4:168-72. 